\title{
EXACT SOLUTION OF THE ASYMMETRIC EXCLUSION MODEL WITH PARTICLES OF ARBITRARY SIZE
}

\author{
F. C. Alcaraz \\ Departamento de Física, Universidade Federal de São Carlos, 13565-905, São Carlos, SP Brazil \\ R. Z. Bariev \\ Departamento de Física, Universidade Federal de São Carlos, 13565-905, São Carlos, SP Brazil \\ The Kazan Physico-Technical Institute of the Russian Academy of Sciences, Kazan 420029, \\ Russia
}

\begin{abstract}
A generalization of the simple exclusion asymmetric model is introduced. In this model an arbitrary mixture of molecules with distinct sizes $s=0,1,2, \ldots$, in units of lattice space, diffuses asymmetrically on the lattice. A related surface growth model is also presented. Variations of the distribution of molecules's sizes may change the excluded volume almost continuously. We solve the model exactly through the Bethe ansatz and the dynamical critical exponent $z$ is calculated from the finite-size corrections of the mass gap of the related quantum chain. Our results show that for an arbitrary distribution of molecules the dynamical critical behavior is on the Kardar-Parizi-Zhang (KPZ) universality.
\end{abstract}

To appear in Phys. Rev. E (1999).

PACS number(s): 02.50.Ey,05.70.Ln,64.60.Ht

Typeset using REVTEX 


\section{INTRODUCTION}

The asymmetric simple exclusion model [1] is a one-dimensional stochastic model that describes the time fluctuations of particles diffusing asymmetrically on the lattice. If we interprete an occupied site as $\sigma_{i}^{z}=+1$ and a vacant site as $\sigma_{i}^{z}=-1$ the time evolution of the

probability distribution of particles is given by the following asymmetric XXZ Hamiltonian

$$
H=-\sum_{i=1}^{N}\left[\epsilon_{+} \sigma_{j}^{-} \sigma_{j+1}^{+}+\epsilon_{-} \sigma_{j}^{+} \sigma_{j+1}^{-}+\frac{1}{4}\left(1-\sigma_{j}^{z} \sigma_{j+1}^{z}\right)\right],
$$

where $N$ is the number of lattice sites and $\sigma_{i}^{ \pm}=\left(\sigma^{x} \pm i \sigma^{y}\right) / 2$ are the raising and lowering Pauli operators. Periodic boundary conditions are imposed and $\epsilon_{+}$and $\epsilon_{-}\left(\epsilon_{+}+\epsilon_{-}=1\right)$ are the transition probabilities for having a motion to the right and to the left, respectively. The physical properties of this non-hermitian quantum chain as well as the related asymmetric six-vertex model are still under extensive investigations [2 4]. This model also describes the surface fluctuations in a growth model known as single-step model [5]6] where $\left(\sigma_{i}^{z}+\right.$ $\left.\sigma_{i+1}^{z}\right) / 2=-1,0,1$ is the height difference between nearest-neighbor steps located at the oddhalf integers sites $(i-1 / 2)(i=1,2, \ldots)$. The master equation defining the Hamiltonian (11) can also be interpreted [7] as the discretized version of the noisy Burges equation or the Kardar-Parisi-Zhang (KPZ) equation [8] governing the motion of the height of growing surfaces whose local growth velocity depends nonlinearly on the local shape. The conection between the scaling behavior of the structure function [6,9] of the stochastic model and the finite-size dependence of the real part of the mass gap $G_{N}$ gives us the dynamical critical exponent $z$,

$$
\operatorname{Re}\left(G_{N}\right) \sim N^{-z}
$$

This connection enabled Gwa and Spohn [0] to explore the Bethe ansatz solution of (11) and calculate exactly the exponent $z=3 / 2$ in the highly anisotropic limit $\epsilon_{-}=0$. Subsequently Kim [10] extended this result for $\epsilon_{-}>0$.

In a previous paper [11] we observed, in connection with a model for strongly correlated system, that it is possible to keep the exact integrability of the XXZ chain by enlarging 
the excluded volume to the spins. Motivated by these results we introduce in this paper a generalization of the simple exclusion model where each particle individually instead of having size 1, in units of lattice spacing, may have an arbitrary and distinct integer size $\left(s_{i}=0,1,2, \ldots\right)$. Particles with size $s_{i}>1$ will produce stronger excluded volume than those in the simple exclusion model where all the particles have unity size $\left(s_{1}=s_{2}=\right.$ $\left.\cdots=s_{n}=1\right)$. Particles with size zero $(s=0)$ produce no excluded volume since we may put an arbitrary number of them at the same site. By considering arbitrary mixtures of molecules with appropriate sizes we may change continuously the excluded volume in the bulk limit. The time-evolution operator for these models are generalizations of ferromagnetic XXZ chain where restrictions on spin configurations, which depend on the particular sizes of the molucules $s_{i}$, are added. We show that for arbitrary distribution of molecule's sizes the eigenspectrum of the related Hamiltonian can be calculated exactly through the Bethe ansatz. The exact integrability for the particular case where all the molecules have the same size and the diffusion is fully asymmetric was also verified recently by Sasamoto and Wadati [12.

Following Gwa and Spohn [7] we show, in the anisotropic limit $\epsilon_{-}=0$, that for arbitrary distribution of molecules the real part of the gap behaves as $\operatorname{Re}(G) \sim N^{-3 / 2}$, giving a universal KPZ behaviour with dynamical critical exponent $z=3 / 2$. Since in our model the excluded volume can be controlled continuously by changing the distribution of molecules, the above exact results imply that the exclusion volume effect is irrelevant to the KPZ dynamics.

The paper is organized as follows. In the next section we introduce the generalized asymmetric model and the related generalized surface growth model. The Bethe-ansatz solution of our model is presented in section 3 and in section 4 a numerical and analitical calculation of the dynamical critical exponent $z$ is presented. Finally in section 5 we give our conclusions and in an appendix we relate exactly the general asymmetric exclusion model with several boundary conditions with the simple exclusion model, in different lattice sizes. 


\section{THE GENERALIZED ASYMMETRIC EXCLUSION MODEL}

The simplest realization of the model we consider in this paper is the asymmetric diffusion of molecules (or particles) on a lattice of size $N$, where each molecules $i(i=1,2, \ldots, n)$ may have a distinct size $s_{i}=0,1,2, \ldots$, in units of lattice spacing. We represent the molecules

on the lattice by placing their center of mass at the lattice sites. In Fig. 1 we show some examples of configurations of $n=5$ molecules with size's distribution $\{s\}$ in a lattice with $N=6$ sites. Molecules of size $s=0$ are special since we can put in a given lattice point an arbitrary number of them. As we can see from Fig. 1, the minimum distance between two particles with size $s, s^{\prime}$ on the lattice is given by

$$
d_{s, s^{\prime}}=\operatorname{Int}\left[\frac{s+s^{\prime}+1}{2}\right], \quad s, s^{\prime}=0,1,2, \ldots,
$$

where $\operatorname{Int}(x)$ is the integer part of $x$. In order to describe the occupancy of a given site $i$ $(i=1,2, \ldots, N)$ we attach on it a site variable $\beta_{i}$ taking integer values $\left(\beta_{i} \in \mathrm{Z}\right)$. If $\beta_{i}=0$ the site is empty, on the other hand if $\beta_{i}>0$ we have a molecule of size $s=\beta_{i}$ and if $\beta_{i}=-n<0$ we have $n$ molecules of size 0 . The allowed configurations $\left\{\beta_{i}\right\}=\left\{\beta_{1}, \beta_{2}, \ldots, \beta_{N}\right\}$ are those satisfying the constraints imposed by the sizes of molecules in a periodic lattice, i.e., if $\beta_{i} \neq 0$ and $\beta_{j} \neq 0$ we must have $|i-j| \geq d_{s\left(\beta_{i}\right), s\left(\beta_{j}\right)}$, where $s(\beta)=0$ if $\beta \leq 0$, and $s(\beta)=\beta$ if $\beta>0$, is the excluded volume (or size) associated to $\beta$.

The master equation for the probability distribution $P(\{\beta\}, t)$ can be written in general as

$$
\frac{\partial P(\{\beta\}, t)}{\partial t}=-\Gamma\left(\{\beta\} \rightarrow\left\{\beta^{\prime}\right\}\right) P(\{\beta\}, t)+\Gamma\left(\left\{\beta^{\prime}\right\} \rightarrow\{\beta\}\right) P\left(\left\{\beta^{\prime}\right\}, t\right)
$$

where $\Gamma\left(\{\beta\} \rightarrow\left\{\beta^{\prime}\right\}\right)$ is the transition rate, where a configuration $\{\beta\}$ changes to $\left\{\beta^{\prime}\right\}$. In the model under consideration we only allow, whenever it is possible, a single particle to diffuse into its nearest neighbor sites. The possible motions are diffusion to the right

$$
\begin{aligned}
& \beta_{i} \emptyset_{i+1} \rightarrow \emptyset_{i} \beta_{i+1}, \quad \beta>0 \\
& \beta_{i} \gamma_{i+1} \rightarrow(\beta+1)_{i}(\gamma-1)_{i+1}, \quad \beta<0, \gamma \leq 0,
\end{aligned}
$$


with transition rate $\epsilon_{R}$ and diffusion to the left

$$
\begin{aligned}
& \emptyset_{i} \beta_{i+1} \rightarrow \beta_{i} \emptyset_{i+1}, \quad \beta>0 \\
& \gamma_{i} \beta_{i+1} \rightarrow(\gamma-1)_{i}(\beta+1)_{i+1} \quad \beta<0, \quad \gamma \leq 0,
\end{aligned}
$$

with transition rate $\epsilon_{L}$. The master equation (2) can be written as a Schrödinger equation in Euclidean time (see ref. [13] for general application for two-body processes)

$$
\frac{\partial \mid P>}{\partial t}=-H \mid P>
$$

if we interpret $\mid P>\equiv P(\{\beta\}, t)$ as the associated wave function. If we represent $\beta_{i}$ as $\mid \beta>_{i}$ the vector $\left|\beta>_{1} \otimes\right| \beta>_{2} \otimes \cdots \otimes \mid \beta>_{N}$ will give us the associated Hilbert space. The process (田) and (5) gives us the Hamiltonian

$$
\begin{aligned}
H & =-D P \sum_{i=1}^{N}\left(H_{i, i+1}^{>}+H_{i, i+1}^{<}\right) P, \\
H_{i, j}^{>} & =\sum_{\beta=1}^{\infty}\left[\epsilon_{+}\left(E_{i}^{0, \beta} E_{j}^{\beta, 0}-E_{i}^{\beta, \beta} E_{j}^{0,0}\right)+\epsilon_{-}\left(E_{i}^{\beta, 0} E_{j}^{0, \beta}-E_{i}^{0,0} E_{j}^{\beta, \beta}\right)\right], \\
H_{i, j}^{<} & =\sum_{\beta=-\infty}^{-1} \sum_{\gamma=-\infty}^{0}\left[\epsilon_{+}\left(E_{i}^{\beta+1, \beta} E_{j}^{\gamma-1, \gamma}-E_{i}^{\beta, \beta} E_{j}^{\gamma, \gamma}\right)+\epsilon_{-}\left(E_{i}^{\gamma-1, \gamma} E_{j}^{\beta+1, \beta}-E_{i}^{\gamma, \gamma} E_{j}^{\beta, \beta}\right)\right],
\end{aligned}
$$

with

$$
D=\epsilon_{R}+\epsilon_{L}, \quad \epsilon_{+}=\frac{\epsilon_{R}}{\epsilon_{R}+\epsilon_{L}}, \quad \epsilon_{-}=\frac{\epsilon_{L}}{\epsilon_{R}+\epsilon_{L}}
$$

and periodic boundary conditions. The matrices $E^{\alpha, \beta}$ are infinite-dimensional with a single non-zero element $\left(E^{\alpha, \beta}\right)_{i, j}=\delta_{\alpha, i} \delta_{\beta, j}(\alpha, \beta, i, j \in \mathrm{Z})$. The projector $\mathrm{P}$ keeps on the Hilbert space only the vectors $\mid\{\beta\}>$ satisfying the constraint (2) which mathematically means that for the all $\beta_{i}, \beta_{j} \neq 0, \quad|i-j| \geq d_{s\left(\beta_{i}\right), s\left(\beta_{j}\right)}$. The constant D in (17) fixes the time scale and for simplicity we chose $D=1$. A particular simplification of the above Hamiltonian occurs when all the molecules have the same fixed size $s>0$. In this case the Hamiltonian can be expressed in terms of spin- $\frac{1}{2}$ Pauli matrices

$$
H_{\left\{s_{1}=\cdots=s_{n}=s\right\}}=-P_{s}\left\{\sum_{i=1}^{N}\left[\epsilon_{+} \sigma_{i}^{-} \sigma_{i+1}^{+}+\epsilon_{-} \sigma_{i}^{+} \sigma_{i+1}^{-}+\frac{1}{2}\left(\epsilon_{+}+\epsilon_{-}\right)\left(\sigma_{i}^{z} \sigma_{i+1}^{z}-1\right)\right]\right\} P_{s},
$$


where now $P_{s}$ projects out configurations where two up spins, in $\sigma^{z}$ basis, are at distance smaller than the size $s>0$. In the case where $s=1$ the projector $P_{s}=1$ and we have the standard asymmetric exclusion Hamiltonian (1). In terms of Pauli matrices this operator has the general form

$$
P_{s}=\prod_{i}\left[\frac{1}{2}\left(1-\sigma_{i}^{z}\right)+\frac{1}{2}\left(1+\sigma_{i}^{z}\right) \prod_{l=1}^{s-1}\left(\frac{1-\sigma_{i+l}^{z}}{2}\right)\right] .
$$

The Hamiltonian (9) can be more easily compared with standard magnetic quantum chains by performing for $\epsilon_{+}, \epsilon_{-} \neq 0$ the following canonical change of variables

$$
\sigma_{i}^{ \pm} \rightarrow\left(\frac{\epsilon_{-}}{\epsilon_{+}}\right)^{ \pm i / 2} \sigma_{i}^{ \pm}, \quad \sigma^{z} \rightarrow \sigma^{z}, \quad i=1,2, \ldots, N
$$

which gives

$$
\begin{aligned}
& H=-\frac{1}{2} \sqrt{\epsilon_{+} \epsilon_{-}} \sum_{i=1}^{N} P_{s}\left[\sigma_{i}^{x} \sigma_{i+1}^{x}+\sigma_{i}^{y} \sigma_{i+1}^{y}+\Delta\left(\sigma_{i}^{z} \sigma_{i+1}^{z}-1\right)\right] P_{s} \\
& \Delta=\frac{\epsilon_{+}+\epsilon_{-}}{\sqrt{\epsilon_{R} \epsilon_{L}}} .
\end{aligned}
$$

Apart from the projector $P_{s}$ this is the ferromagnetic XXZ chain or the anisotropic Heisenberg chain. However in distinction with (8) the boundary conditions are now twisted

$$
\sigma_{N+1}^{ \pm}=\left(\frac{\epsilon_{+}}{\epsilon_{-}}\right)^{ \pm N / 2} \sigma_{1}^{ \pm}, \quad \sigma_{N+1}^{z}=\sigma_{1}^{z}
$$

We expect that ferromagnetic quantum chains like those in (12) are gapped for $\Delta>1$ However since $\left(\epsilon_{+} / \epsilon_{-}\right)^{N / 2} \rightarrow \infty$, for $N \rightarrow \infty$ the boundary condition gives us interaction with the same degree of importance as the totality of the other interactions (see [14 for a related problem). As we will see, from the exact solution of (7) and (9), this surface term is strong enough to produce a gapless eigenspectrum.

In surface growth physics the asymmetric simple exclusion model is related to the singlestep model. Similarly our generalized model is also related to a generalization of the singlestep model. The surface configurations in this growth model are obtained by defining height variables $h_{i}(i=1,2, \ldots)$ which are related to the spin variables in our generalized asymmetric diffusion model. For simplicity we are going to present only the surface growth model 
related to the diffusion problem where all molecules have the same size $s$. Let us consider initially $s>0$. For a given configuration $\left\{\beta_{1}, \ldots, \beta_{N}\right\}$ of molecules of size $s$, the height variables should obey

$$
h_{i+1}-h_{i}=f\left(\beta_{i-\frac{s}{2}}, \beta_{i-\frac{s}{2}+1}, \ldots, \beta_{i+\frac{s}{2}}, \beta_{i+\frac{s}{2}+1}\right) \text {, }
$$

where $f=0$ for all allowed configurations except in the case

$$
f(0, \ldots, 0)=-1 \quad \text { and } \quad f(1,0, \ldots, 0,1)=1
$$

The variables $\{\beta\}$ of the related diffusion model are defined at the links or at the same positions of the height variables $\{h\}$ depending if the size of the molecules $s$ is odd or even, respectively. The number of molecules $n(0,1, \ldots)$, in the generalized asymmetric diffusion is conserved and for each value of $n$ we are going to have a growth model with different boundary conditions in the spacial direction. The dynamical rules defining the growth are the following

a) No steps on the surface are allowed to be higger than 1, in units of lattice spacing in the growth direction, i. e.,

$$
h_{i+1}-h_{i}=1,0,-1 \quad(i=1, \ldots, n-1) .
$$

b) All the local valleys and hills should have a size, in units of the lattice spacing in the spatial direction, which is a multiple of $b=s+1$.

c) The following boundary condition should be satisfied

$$
h_{i+N}=h_{i}-\bar{h}, \quad \bar{h}=b\left\{[N / b]_{I}-n\right\}+[N / b]_{R} .
$$

where by $[N / b]_{I}$ and $[N / b]_{R}$ we mean the integer part and the rest of the division $N / b$.

d) The surface changes whenever obeying the previous requirements we can still adsorb $\left(h_{i+l} \rightarrow h_{i+l}+1, l=0,1, \ldots, b-1\right)$ or desorb $\left(h_{i+l} \rightarrow h_{i+l}-1, l=0,1, \ldots, b-1\right)$ at height $h_{i}$ $(i=1, \ldots, N)$ a retangular brick of size $b$ in the spatial direction and size 1 in the growth direction. 
We choose a height $h_{i}(i=1, \ldots, n)$ at random. If it is possible to adsorb or desorb a brick, with probability $\epsilon_{+} / 2\left(\epsilon_{-} / 2\right)$ we desorb (adsorb) a brick, and do nothing with probability $\frac{1}{2}$. If it is possible, at $h_{i}$, only to desorb (adsorb) a brick, with probability $\epsilon_{+}\left(\epsilon_{-}\right)$we desorb (adsorb) a brick and with probability $1-\epsilon_{+}\left(1-\epsilon_{-}\right)$we do nothing. In Fig. 2 we show for $N=7, s=2(b=3)$ and $n=2$ some examples of the possible configurations of the surface. In this figure we also show the corresponding particle configurations in the diffusion problem. We can verify that for arbitrary $s$ (or b), as long the growth model is not periodic $(N \neq n b)$ there exists an exact one-to-one correspondence between the configurations of particles and those of the surface, with the transitions between them described by the Hamiltonian (7). On the other hand, if the growth model is periodic $(N=n b)$, there exist $b$ configurations in the asymmetric diffusion problem that correspond to a single surface configuration (the flat surface). Consequently the Hamiltonian (7) does not describe exactly the generalized step model in a finite lattice. However as $N$ increases this difference decreases and (7) also describes the fluctuations of the growth model.

Finally, in the case where all the molecules has size zero a possible growth model is obtained by defining height variable $h_{i}(i=1,2, \ldots, N)$ at the same position of the molecules in the diffusion problem. For a given configuration $\left\{n_{1}, n_{2}, \ldots, n_{N}\right\}$ with $n_{i}$ molecules at sites $i$ the height variables in the surface model $\left(h_{i+1} \geq h_{i}\right)$ satisfy

$$
h_{i}-h_{i-1}=n_{i}, \quad i=2, \ldots, N \text {, }
$$

with the boundary condition

$$
h_{N+1}=h_{1}+n
$$

where $n=\sum n_{i}$ is the total number of molecules. Bricks of unity size are desorbed (adsorbed) with transition rates $\epsilon_{+}\left(\epsilon_{-}\right)$if the final configuration satisfies $h_{i+1} \geq h_{i}(i=1,2, \ldots, N-1)$. 


\section{THE BETHE ANSATZ EQUATIONS}

We present in this section the exact solution of the general quantum chain (7). For simplicity let us consider initially the case where all the molecules have the same size $s$ $(0,1, \ldots)$. In the particular case where $s=1$ we have the standard simple exclusion model whose Bethe ansatz solution was obtained by Gwa and Spohn [7] and can also be obtained after the canonical transformation (13) from the Bethe ansatz solution of the XXZ chain with twisted boundary conditions [15]. The exact integrability of the fully asymmetric version of (12) $\left(\epsilon_{-}=0\right)$, for $s>0$, was verified directly in the master equation by Sasamoto and Wadati [12], and the model with $s=0$ is related to the limit $q \rightarrow \infty$ of the $q$-boson hopping model introduced by Bogoliubov et al. [16,17].

Due to the conservation of particles in the diffusion processes the total number of particles are good quantum numbers and we can separate the associated Hilbert space into blockdisjoint sectors labelled by the number $n$ of particles. We therefore consider the eigenvalue equation

$$
H|n>=E| n>
$$

where

$$
\left|n>=\sum_{\{x\}} f\left(x_{1}, \ldots, x_{n}\right)\right| x_{1}, \ldots, x_{n}>
$$

Here $x_{1}, \ldots, x_{n}$ denotes the location of particles on the chain and the summation extends over all sets $\{x\}$ of the $n$ non-decreasing integers satisfying

$$
x_{i} \geq x_{i+1}+s, \quad i=1, \ldots, n-1, \quad s \leq x_{n}-x_{1} \leq N-s
$$

It is important to notice that some of these coordinates may coincide in the case where the particles have zero size.

$\mathbf{n}=\mathbf{1}$. For one particle on the chain as a consequence of the translational invariance of (घ) the eigenfunctions are the momentum- $k$ eigenfunctions 


$$
\left|1>=\sum_{x=1}^{N} f(x)\right| x>, \quad f(x)=e^{i k x}
$$

where

$$
k=\frac{2 \pi}{N} l ; \quad l=0,1, \ldots, N-1
$$

and energy given by

$$
E=e(k) \equiv-\left(\epsilon_{-} e^{i k}+\epsilon_{+} e^{-i k}-1\right)
$$

$\mathbf{n}=\mathbf{2}$. For two particles on the lattice the eigenvalue equation (20) gives us two distinct relations depending on the relative position of the particles. If the two particles are at positions $x_{1}$ and $x_{2}$ satisfying $x_{2}>x_{1}+s$ we obtain

$$
\begin{aligned}
E f\left(x_{1}, x_{2}\right)= & -\epsilon_{+} f\left(x_{1}-1, x_{2}\right)-\epsilon_{-} f\left(x_{1}+1, x_{2}\right)+2 f\left(x_{1}, x_{2}\right) \\
& -\epsilon_{+} f\left(x_{1}, x_{2}-1\right)-\epsilon_{-} f\left(x_{1}, x_{2}+1\right),
\end{aligned}
$$

that can be solved by the ansatz

$$
f\left(x_{1}, x_{2}\right)=e^{i k_{1} x_{1}} e^{i k_{2} x_{2}}
$$

which gives

$$
E=e\left(k_{1}\right)+e\left(k_{2}\right)
$$

Since the relation (28) is symmetric in $k_{1}$ and $k_{2}$ we can write a more general solution of (26) as

$$
f\left(x_{1}, x_{2}\right)=A_{12} e^{i k_{1} x_{1}} e^{i k_{2} x_{2}}+A_{21} e^{i k_{2} x_{1}} e^{i k_{1} x_{2}}
$$

with the same energy as in (28). The second relation happens when $x_{2}=x_{1}+s$. In this case we have

$$
E f\left(x_{1}, x_{1}+s\right)=-\epsilon_{+} f\left(x_{1}-1, x_{1}+s\right)-\epsilon_{-} f\left(x_{1}, x_{1}+s+1\right)+f\left(x_{1}, x_{1}+s\right) .
$$


If we now substitute the ansatz (29) with the energy (28), the constants $A_{12}$ and $A_{21}$, initially arbitrary, should now satisfy

$$
\begin{gathered}
\frac{A_{12}}{A_{21}}=-\left(\frac{e^{i k_{1}}}{e^{i k_{2}}}\right)^{s-1} e^{i \Psi_{12}}, \\
e^{i \Psi_{j l}}=\frac{\epsilon_{+}+\epsilon_{-} e^{i\left(k_{j}+k_{l}\right)}-e^{i k_{j}}}{\epsilon_{+}+\epsilon_{-} e^{i\left(k_{j}+k_{l}\right)}-e^{i k_{l}}} .
\end{gathered}
$$

The "wave numbers" $k_{1}$ and $k_{2}$ are complex in general and are fixed due to the cyclic boundary condition

$$
f\left(x_{2}, x_{1}+N\right)=f\left(x_{1}, x_{2}\right)
$$

which from (29) give us the equations

$$
\frac{A_{12}}{A_{21}}=e^{-i k_{2} N}, \quad \frac{A_{21}}{A_{12}}=e^{-i k_{1} N}
$$

Equations (31) and (32) give us the Bethe-ansatz equations for $n=2$

$$
e^{i k_{j} N}=-\prod_{l=1}^{2}\left(\frac{e^{i k_{j}}}{e^{i k_{l}}}\right)^{s-1} e^{i \Psi_{j, l}}, \quad j=1,2,
$$

with energy given by (28).

General n. The above calculation can be generalized for arbitrary values of $n$. The ansatz for the wavefunction becomes

$$
f\left(x_{1}, \ldots, x_{n}\right)=\sum_{P} A_{P_{1}, P_{2}, \ldots, P_{n}} e^{i\left(k_{P_{1}} x_{1}+\cdots+k_{P_{n}} x_{n}\right)},
$$

where the sum extends over all permutations $P$ of $1,2, \ldots, n$. If $x_{i+1}-x_{i}>s$ for $i=$ $1,2, \ldots, n$ it is easy to see that the eigenvalue equation (20) is satisfied by the ansatz (36) with energy

$$
E=\sum_{j=1}^{n} e\left(k_{j}\right)
$$

If a pair of paticles is at positions $x_{i}, x_{i+1}$, where $x_{i+1}=x_{i}+s$, equation (20) with the ansatz (36) and the relation (37) give us 


$$
\frac{A_{P 1, \ldots, P_{i}, P_{i+1}, \ldots, P_{n}}}{A_{P_{1}, \ldots, P_{i+1}, P_{i}, \ldots, P_{n}}}=-e^{i(s-1)\left(k_{P_{i}}-k_{P_{i+1}}\right)} e^{i \Psi_{P_{i}, P_{i+1}}} .
$$

Inserting the ansatz (36) in the boundary condition

$$
f\left(x_{2}, \ldots, x_{n}, x_{1}+N\right)=f\left(x_{1}, \ldots, x_{n}\right)
$$

we obtain the additional relation

$$
A_{P_{1}, \ldots, P_{n}}=e^{i k_{P_{1}} N} A_{P_{2}, \ldots, P_{n}, P_{1}}
$$

If we iterate the relation (38) $n$ times the equation (40) gives us the Bethe-ansatz equations

$$
e^{i k_{j} N}=(-)^{n-1} \prod_{l=1}^{n}\left(\frac{e^{i k_{j}}}{e^{i k_{l}}}\right)^{s-1} \frac{\epsilon_{+}+\epsilon_{-} e^{i\left(k_{j}+k_{l}\right)}-e^{i k_{j}}}{\epsilon_{+}+\epsilon_{-} e^{i\left(k_{j}+k_{l}\right)}-e^{i k_{l}}}
$$

for $j=1,2, \ldots, n$. The solutions $\left\{k_{j}\right\}$ of these equations with (37) give us the eigenergies of the Hamiltonian (7). Furthermore, it follows from a lattice shifting that the wavefunctions given by the ansatz (36) are also eigenfunctions of the momentum operator $\hat{P}$ with eigenvalue

$$
p=\sum_{j=1}^{n} k_{j} \quad \bmod (2 \pi)=\frac{2 \pi}{N} l, \quad l=0,1, \ldots, N-1 .
$$

In the particular case where $s=1$ equations (37),(41) and (42) recover the results presented in Ref. [7].

Let us now consider the general case where we have $n$ molecules with arbitrary given sizes $\left\{s_{1}, s_{2}, \ldots, s_{n}\right\}\left(s_{i}=0,1,2, \ldots\right)$ and the Hamiltonian given by (7). In this case each type of molecule is conserved separately. Moreover since in the diffusion processes the particles only interchange positions with the vacant sites a given order $\left\{s_{1}, s_{2}, \ldots, s_{n}\right\}$ of particles remain conserved up to cyclic permutations. The wavefunctions can be written as

$$
\left|s_{1}, s_{2}, \ldots, s_{n}>=\sum_{\{c\}} \sum_{\{x\}} f^{s_{c_{1}}, \ldots, s_{c_{n}}}\left(x_{1}, \ldots, x_{n}\right)\right| x_{1}, \ldots, x_{n}>
$$

Here $f^{s_{c_{1}}, \ldots, s_{c_{n}}}\left(x_{1}, \ldots, x_{n}\right)$ is the amplitude of a configuration where particles of sizes $s_{1}, \ldots, s_{n}$ occupy the positions $x_{1}, \ldots, x_{n}$, respectively. The summation $\{c\}$ extends over all cyclic permutations $\left\{c_{1}, \ldots, c_{n}\right\}$ of integers $\{1, \ldots, n\}$, and the summation $\{x\}$ extends, for a given distribution $\left\{s_{c_{1}}, \ldots, s_{c_{n}}\right\}$ of molecules, to increasing integers satisfying 


$$
x_{i+1} \geq x_{i}+d_{s_{c_{i}}, s_{c_{i+1}}} \quad i=1, \ldots, n-1, \quad d_{s_{c_{n}}, s_{c_{1}}} \leq x_{n}-x_{1} \leq N-d_{s_{c_{n}}, s_{c_{1}}} .
$$

The ansatz we expect to be valid, that replaces (35) is

$$
f^{s_{1}, \ldots, s_{n}}\left(x_{1}, \ldots, x_{n}\right)=\sum_{P} A_{P_{1}, \ldots, P_{n}}^{s_{1}, \ldots, s_{n}} e^{i\left(k_{P_{1}}+\cdots+k_{P_{n}}\right)}
$$

where $A_{k_{P_{1}}, \ldots, k_{P_{n}}}^{s_{1}, \ldots, s_{n}}$ and $\left\{k_{1}, \ldots, k_{n}\right\}$ are going to be fixed by imposing that (44) with (45) is a solution of the eigenvalue equation of the general Hamiltonian (7).

Let us consider the eigenvectors of (1) with different number of particles.

$\mathbf{n}=\mathbf{1}$. For one particle on the chain the ansatz (45) coincides with (36) and the wavefunctions and energies are given by (23) and (25), respectively.

$\mathbf{n}=2$. If both particles are identical $s_{1}=s_{2}=s$, we have the same situation considered previously in (26)-(35). The wavefunctions $\mid s, s>$ are given by (45) with energies given by (28) and (35). However, if the particles are distinct the situation is different. If the particles are located at positions $x_{1}$ and $x_{2}$, with $x_{2}-x_{1}>d_{s_{1}, s_{2}}$ the ansatz (44) is valid with energy given by (28) and no restrictions on $\left\{A_{k_{P_{1}}, k_{P_{2}}}^{\alpha_{i}, \alpha_{j}}\right\}$ are necessary. If the particles are at the closest distance $x_{2}=x_{1}+d_{s_{1}, s_{2}}$ equation (30) is replaced by

$$
\begin{aligned}
E f^{s_{1}, s_{2}}\left(x_{1}, x_{1}+d_{s_{1}, s_{2}}\right)= & -\epsilon_{+} f^{s_{1}, s_{2}}\left(x_{1}-1, x_{1}+d_{s_{1}, s_{2}}\right)-\epsilon_{-} f^{s_{1}, s_{2}}\left(x_{1}, x_{1}+d_{s_{1}, s_{2}}+1\right) \\
& +f^{s_{1}, s_{2}}\left(x_{1}, x_{1}+d_{s_{1}, s_{2}}\right) .
\end{aligned}
$$

Inserting in the above equation the ansatz (45) and the energy (28) we obtain the relation

$$
A_{P_{1}, P_{2}}^{s_{1}, s_{2}}=-e^{i \Psi_{P_{1}, P_{2}}} \sum_{s_{1}^{\prime}, s_{2}^{\prime}} S_{s_{2}^{\prime}, s_{1}^{\prime}}^{s_{1}, s_{2}}\left(k_{P_{1}}-k_{P_{2}}\right) A_{P_{2}, P_{1}}^{s_{1}^{\prime}, s_{2}^{\prime}}
$$

where $\Psi_{P_{1}, P_{2}}$ is given by (32) and the elements of the $S$ matrix are given by

$$
S_{\gamma, \mu}^{\alpha, \beta}(k)=e^{i\left(d_{\alpha, \beta}-1\right) k} \delta_{\alpha, \mu} \delta_{\beta, \gamma}
$$

The wave numbers $k_{1}$ and $k_{2}$ are going to be fixed by the boundary condition

$$
f^{s_{2}, s_{1}}\left(x_{2}, x_{1}+N\right)=f^{s_{1}, s_{2}}\left(x_{1}, x_{2}\right),
$$


but instead of deriving the Bethe-ansatz equations for $n=2$ let us consider the case of general $n$.

General n. The ansatz (45) applied to the case where two particles are at their closest distance gives us the generalization of (47)

$$
A_{\ldots, P_{1}, P_{2}, \ldots}^{\ldots, \alpha, \beta, \ldots}=-e^{i \Psi_{P_{1}, P_{2}}} \sum_{\alpha^{\prime}, \beta^{\prime}} S_{\alpha^{\prime}, \beta^{\prime}}^{\alpha, \beta}\left(k_{P_{1}}-k_{P_{2}}\right) A_{\ldots, P_{2}, P_{1}, \ldots}^{\ldots, \beta^{\prime}, \alpha^{\prime}, \ldots}
$$

with $S$ given by (48). Successive applications of this equation give us in general different relations between the amplitudes. For example $A_{\ldots, k_{1}, k_{2}, k_{3}, \ldots}^{\ldots, \alpha, \beta, k_{1}}$ relate to $A_{\ldots, k_{3}, k_{2}, k_{1}, \ldots}^{\ldots, \gamma, \beta, \alpha}$ by performing the permutations $\alpha \beta \gamma \rightarrow \beta \alpha \gamma \rightarrow \beta \gamma \alpha \rightarrow \gamma \beta \alpha$ or $\alpha \beta \gamma \rightarrow \alpha \gamma \beta \rightarrow \gamma \alpha \beta \rightarrow \gamma \beta \alpha$, and consequently the $S$-matrix should satisfy the Yang-Baxter [18,19] equation

$$
\begin{gathered}
\sum_{\gamma, \gamma^{\prime}, \gamma^{\prime \prime}} S_{\gamma, \gamma^{\prime}}^{\alpha, \alpha^{\prime}}\left(k_{1}-k_{2}\right) S_{\beta, \gamma^{\prime \prime}}^{\gamma, \alpha^{\prime \prime}}\left(k_{1}-k_{3}\right) S_{\beta^{\prime}, \beta^{\prime \prime}}^{\gamma^{\prime}, \gamma^{\prime \prime}}\left(k_{2}-k_{3}\right)= \\
\sum_{\gamma, \gamma^{\prime}, \gamma^{\prime \prime}} S_{\gamma^{\prime}, \gamma^{\prime \prime}}^{\alpha^{\prime}, \alpha^{\prime \prime}}\left(k_{2}-k_{3}\right) S_{\gamma, \beta^{\prime \prime}}^{\alpha, \gamma^{\prime \prime}}\left(k_{1}-k_{3}\right) S_{\beta, \beta^{\prime}}^{\gamma, \gamma^{\prime}}\left(k_{1}-k_{2}\right) .
\end{gathered}
$$

Actually the relation (51) is a necessary and sufficient condition [18,19] to obtain non-trivial solution for the amplitudes in (50). The validity of (51) can easily be verified for the diagonal $S$-matrix (48) in the problem we are considering.

The boundary condition

$$
f^{s_{1}, \ldots, s_{n}}\left(x_{1}, \ldots, x_{n}\right)=f^{s_{2}, \ldots, s_{n}, s_{1}}\left(x_{2}, \ldots, x_{n}, x_{1}\right)
$$

implies the relation between the amplitudes

$$
A_{P_{1}, \ldots, P_{n}}^{s_{1}, \ldots, s_{n}}=e^{i k_{P_{1}} N} A_{P_{2}, \ldots, P_{n}, P_{1}}^{s_{2}, \ldots, s_{n}, s_{1}}
$$

If we now apply relation (50) $n$ times we can obtain a relation between the amplitudes with same momenta, i. e.,

$$
\begin{aligned}
& A_{P_{1}, \ldots, P_{n}}^{s_{1}, \ldots, s_{n}}=(-)^{n-1} e^{i \sum_{l=2}^{n} \Psi_{P_{l}, P_{1}}} e^{i k_{P_{1}} N} \\
& \sum_{\left\{s_{1}^{\prime}, \ldots, s_{n}^{\prime}\right\}} \sum_{\left\{s_{1}^{\prime \prime}, \ldots, s_{n}^{\prime \prime}\right\}} S_{s_{1}^{\prime}, s_{1}^{\prime \prime}}^{s_{1}, s_{2}^{\prime \prime}}\left(k_{P_{1}}-k_{P_{1}}\right) S_{s_{2}^{\prime}, s_{2}^{\prime \prime}}^{s_{2}, s_{3}^{\prime \prime}}\left(k_{P_{2}}-k_{P_{1}}\right) \cdots S_{s_{n}^{\prime}, s_{n}^{\prime \prime}}^{s_{n}, s_{1}^{\prime \prime}}\left(k_{P_{n}}-k_{P_{1}}\right) A_{P_{1}, \ldots, P_{n}}^{s_{1}^{\prime}, \ldots, s_{n}^{\prime}},
\end{aligned}
$$

where we introduced the extra sum 


$$
1=\sum_{s_{2}^{\prime \prime}, s_{1}^{\prime \prime}} \delta_{s_{2}^{\prime \prime}, s_{1}^{\prime}} \delta_{s_{1}, s_{1}^{\prime \prime}}=\sum_{s_{2}^{\prime \prime}, s_{1}^{\prime \prime}} S_{s_{1}^{\prime}, s_{1}^{\prime \prime}}^{s_{1}, s^{\prime \prime}}\left(k_{P_{1}}-k_{P_{1}}\right)
$$

In order to fix the values of $\left\{k_{j}\right\}$ we should then find the eigenvalues $\Lambda(k)$ of the matrix

$$
<\{s\}|T(k)|\left\{s^{\prime}\right\}>=\sum_{\left\{s_{1}^{\prime \prime}, \ldots, s_{n}^{\prime \prime}\right\}} \prod_{l=1}^{n} S_{s_{l}^{\prime}, s_{l}^{\prime \prime}}^{s_{l}^{\prime \prime}}\left(k_{P_{l}}-k\right),
$$

with $s_{n+1}^{\prime \prime}=s_{1}^{\prime \prime}$. We identify $T(k)$ as the transfer matrix of a inhomogeneous vertex model, with inhomogeneities $\left(k_{P_{l}}-k\right)$, in a periodic lattice. The Boltzmann weights of the vertex models are given by $S_{\gamma \delta}^{\alpha \beta}$ and the number of distinct vertices depends on the particular configuration (type of order) of molecules in our diffusive system. Using (48) we can see that there exists only one non-zero element for each line, i. e., $<s_{1}, \ldots, s_{n}|T| s_{2}, \ldots, s_{n}, s_{1}>$.

In order to calculate the eigenvalues $\Lambda(k)$ we apply the transfer matrix $r$ times in the state $A^{s_{1}, \ldots, s_{n}}$, where $r$ is the minimum number of cyclic rotations of $\left\{s_{1}, \ldots, s_{n}\right\}$ where the configuration repeats the initial one. We may show that

$$
T^{r} A_{P_{1}, \ldots, P_{n}}^{\alpha_{1}, \ldots, \alpha_{n}}=\Lambda^{r}(k) A_{P_{1}, \ldots, P_{n}}^{\alpha_{1}, \ldots, \alpha_{n}}
$$

Also it is easy to compute

$$
\Lambda^{r}(k)=\exp \left[i \frac{r}{n}\left(\sum_{l=1}^{n} d_{s_{l}, s_{l+1}}-1\right) \sum_{l=1}^{n}\left(k_{l}-k\right)\right]
$$

Finally, substituting $\Lambda\left(k_{P_{1}}\right)$ in (54) we obtain the Bethe-ansatz equations

$$
e^{i k_{j} N}=e^{i \frac{2 \pi}{r} m}(-1)^{n-1} \prod_{l=1}^{n} e^{i\left(k_{j}-k_{l}\right)(\tilde{s}-1)} \frac{\epsilon_{+}+\epsilon_{-} e^{i\left(k_{j}+k_{l}\right)}-e^{i k_{j}}}{\epsilon_{+}+\epsilon_{-} e^{i\left(k_{j}+k_{l}\right)}-e^{i k_{l}}},
$$

where $j=1, \ldots, n ; m=0,1, \ldots, r-1$ and

$$
\tilde{s}=\frac{1}{n} \sum_{l=1}^{n} d_{s_{l}, s_{l+1}}
$$

plays the role of an average molecule size of the particular configuration $\{s\}$ of molecules. As we can see, by comparing (59) with (41) the extra phase $\exp (i 2 \pi m / r)(m=0,1, \ldots, r-1)$ gives $r$ times more solutions of (59) than in (41). This indeed should be the case since the Hilbert space associated to the Hamiltonian (7) of a given distribution of particles of sizes 
$\left\{s_{1}, \ldots, s_{n}\right\}$, due to the distinguibility of the particles, is $r$ times bigger than that of the Hamiltonian (17) when $s_{1}=s_{2}=\cdots=s_{n}$. It is interesting to observe that $\tilde{s}$ can take any non-negative rational number by choosing appropriately $\left\{s_{1}, \ldots, s_{n}\right\}$. Also many distinct distributions of molecule's sizes with the same effective $\tilde{s}$ will have the same eigenenergies.

In an appendix we explore our Bethe-ansatz solution to obtain the relationship between the eigenvalues and eigenvectors of the Hamiltonian (可) with different distributions of molecule's sizes.

\section{THE CRITICAL EXPONENT Z}

In this section we calculate the dynamical critical exponent $z$ for the stochastic models presented in Sec. 2. This calculation is done by exploiting its connection with the mass gap $G_{N}$ of the corresponding Hamiltonian,

$$
\operatorname{Re}\left(G_{N}\right) \sim N^{-z}
$$

A calculation for arbitrary values of $\epsilon_{+}, \epsilon_{-}$and density $n / N$ can be done systematically by using the method presented in [10]. However, since universality arguments indicate that $z$ should be independent on the particular values of $\epsilon_{+}, \epsilon_{-}$and $n$, as long as $\epsilon_{+} \neq \epsilon_{-}$, we are going to restrict ourselves, like in [7], to the simplest case where $\epsilon_{-}=0, \epsilon_{+}=1$. A general discussion for the other cases, which does not change our results is given at the end of the appendix. Defining the variables

$$
z_{j}=2 e^{-i k_{j}}-1
$$

the energies (37) and momenta (42) are given by

$$
\begin{gathered}
E=\sum_{j=1}^{n}\left(1-z_{j}\right) / 2, \\
e^{-i P}=\prod_{j=1}^{n}\left(1+z_{j}\right) / 2,
\end{gathered}
$$


respectively. The $\left\{z_{j}\right\}$ variables should satisfy the Bethe-ansatz equations (59)

$$
\left(1+z_{j}\right)^{N-n \tilde{s}}\left(1-z_{j}\right)^{n}=-2^{N} e^{-i \frac{2 \pi}{r} m} \prod_{l=1}^{n} \frac{z_{l}-1}{\left(z_{l}+1\right)^{\tilde{s}}}
$$

where $j=1, \ldots, n, m=0,1, \ldots, r-1$. It is interesting to note that these equations are simpler than the usual Bethe-ansatz equations appearing in other exact integrable systems since the right-hand side of (65) is independent of the particular value of $j$. These equations are even simpler for the special "half-filled" density $\rho=n / N=1 /(1+\tilde{s})$, i. e.,

$$
\begin{array}{r}
\left(1-z^{2}\right)^{n}=Y \\
Y=-2^{n(1+\tilde{s})} \prod_{l=1}^{n} \frac{z_{l}-1}{\left(z_{l}+1\right)^{\tilde{s}}} .
\end{array}
$$

If we parametrize $Y=-a^{n} e^{i n \theta}$, with $a \geq 0$ and $\theta \in\left(-\frac{\pi}{n}, \frac{\pi}{n}\right)$, the $2 n$ roots $z_{j}$ are given by

$$
\begin{aligned}
& z_{j}=\left(1-y_{j}\right)^{\frac{1}{2}}, \quad z_{j+n}=-z_{j} ; \\
& y_{j}=a e^{i \theta} e^{i 2 \pi\left(j-\frac{1}{2}\right) / n} ; \quad j=1, \ldots, n .
\end{aligned}
$$

For a given choice $\left\{z_{j}\right\}$ of the above set and a given value of $m(0,1, \ldots, r-1)$ we have only two unknowns, $a$ and $\theta$, which are obtained from the equation

$$
\left(a e^{i \theta}\right)^{n}=e^{i \frac{2 \pi}{r} m} 2^{(\tilde{s}+1) n} \prod_{l=1}^{n} \frac{z_{l(j)}-1}{\left[z_{l(j)}+1\right]^{\tilde{s}}} .
$$

We have solved numerically the above equations for several values of $\tilde{s}, m, r$ and $n$. The ground-state energy $E=0$ is obtained by choosing $m=0$ in (68), and is given by the configuration

$$
C_{0}=\left\{z_{1}, z_{2}, \ldots, z_{n}\right\}
$$

with $a=\theta=0$. In order to find the first excited state we should consider different choices of $\left\{z_{j}\right\}$ and different values of $m$. Since $z_{j}+z_{n+j}=0$ the energy increases as we take, in the configurations $\left\{z_{j}\right\}$, values of $z_{j}$ where $n<j \leq 2 n$. Therefore configurations $\{z\}$ associated with low energies are

$$
C_{1}=\left\{z_{1}, z_{2}, \ldots, z_{n-1}, z_{n+1}\right\}
$$


and

$$
C_{-1}=\left\{z_{2}, \ldots, z_{n-1}, z_{n}, z_{2 n}\right\}
$$

These configurations, from (64), correspond to states with momentum $-\frac{2 \pi}{N}$ and $\frac{2 \pi}{N}$, respectively. Our numerical results show that the energy corresponding to the configuration $C_{0}$ with $m \neq 0$ behaves for large $N$ as

$$
E_{C_{0}, m} \sim \frac{a}{n^{\frac{1}{2}}}-i \frac{\pi}{r(\tilde{s}+1)} m \quad m=1,2, \ldots .
$$

On the other hand, the configuration $C_{1}$ or $C_{-1}$, for sufficiently large values of $N$ produces the lowest energy when $m=0$, independently of $\tilde{s}, r$ and behaves as

$$
E_{C_{ \pm 1}, 0} \sim \frac{a_{0}}{n^{z}}+i \frac{b_{0}}{n^{\gamma}}, \quad z=\frac{3}{2}, \quad \gamma=1
$$

where $a_{0}$ and $b_{0}$ are constants. The energies for different values of $m$ but with configurations $C_{ \pm 1}$ also behave similarly as $(\sqrt{73})$. Comparing $(\sqrt{73})$ with $(\sqrt{72})$ we see that the gap is given by $E_{C_{ \pm 1}, 0}$ and is real only for the special case $\tilde{s}=1$, treated in [7]. The values of $a$ and $\theta$ that correspond to the first excited state behaves asymptotically as

$$
a=1+\frac{\beta}{n}+o\left(n^{-1}\right), \quad \theta= \pm \alpha \frac{(\tilde{s}-1)}{n^{\frac{3}{2}}}+o\left(n^{-\frac{3}{2}}\right)
$$

where $\beta$ and $\alpha$ are constants. In order to illustrate our numerical results we show in Table 1 the finite-size estimates for the amplitudes $a_{0}, b_{0}$ and the exponents $z$ and $\gamma$ defined in equation (73).

Accepting the behavior (74) for the values of $a$ and $\theta$ for the first excited state we also used the same procedure as in Gwa and Spohn [7] in order to show analitically that $z=\frac{3}{2}$, $a_{0}=2.30134596 \ldots$, independently of the value of $\tilde{s}$ and $b_{0}=\pi(\tilde{s}-1) /(2(\tilde{s}+1))$. In the last line of Table 1 we show the exact results obtained analitically.

These results indicate that all these models with arbitrary mixture if molecules of different sizes, as well as the corresponding generalized growth models exhibit a universal behavior with a KPZ-type of dynamical behavior. In the appendix we show that for general 
values of $\epsilon_{+}, \epsilon_{-}$and $n$ the wave functions of (7) for arbitrary distributions of molecule's sizes, are exactly related. This implies that conditional probabilities and correlation functions for arbitrary distribution $\{s\}$ are exactly related to the simple exclusion problem $\left\{s_{1}=s_{2}=\cdots s_{n}=1\right\}$. The eigenvalues of these models are exactly related in the case of

free boundaries. In the case we have a periodic lattice the eigenvalues of $H^{\{s\}}$ are exactly related to the asymmetric XXZ chain with twisted boundary condition $\phi$ proportional to the momentum of the first excited state. Since the momentum of this state is $P=2 \pi / N$ the effect of the twisted angle should not affect the leading order in the mass gap calculations. This implies that for arbitrary values of $\epsilon_{+}, \epsilon_{-}$and density $n$ the leading order results of the real part of the gap are the same as those calculated systematically in 10 .

\section{CONCLUDING REMARKS}

We have solved exactly a general asymmetric diffusion problem where the particles may have distinct and arbitrary integer sizes. We also show in Sec. 2 that these diffusion models are related to generalized growth models. Since through diffusion the particles do not interchange positions, a given order $\left\{s_{1}, \ldots, s_{n}\right\}$ of the distribution of molecule's sizes on the lattice is fixed, up to cyclic permutations. A parameter which is proportional to the excluded volume for the particles is the average size of the molecules $\tilde{s}$ given by (60). In the case of the simple exclusion problem all the molecules have the same unity size $s_{1}=s_{2}=\cdots=s_{n}=1$, which gives $\tilde{s}=1$. On the other hand, if all the particles has no size we have $\tilde{s}=0$ and there is no excluded volume. By choosing suitable distribution $\{s\}$ of molecule's sizes we may change $\tilde{s}$ almost continuously in the bulk limit $N \rightarrow \infty$. Exploiting the connection between the dyanmical critical exponent $z$ and the mass gap of the related quantum chain we obtained $z=\frac{3}{2}$ for all the models, independently of the parameter $\tilde{s}$ measuring the excluded volume. This implies that, at least in one dimension, the excluded volume effect is irrelevant for dynamical systems in the KPZ universality class.

We also show (see the appendix) that the wave functions of the models with arbitrary 
distribution of molecule's sizes can be related to those of a simple asymmetric exclusion problem, in a distinct lattice size. This implies that conditional probabilities and correlation functions of these models are exactly related.

\section{ACKNOWLEDGMENTS}

This work was supported in part by Conselho Nacional de Desenvolvimento Científico e Tecnológico - CNPq - Brasil, by FINEP - Brasil and by the Russian Foundation of Fundamental Investigation ( Grant 99-02-17646).

\section{APPENDIX A: EXACT SPECTRAL RELATIONS BETWEEN THE MODELS WITH DISTINCT MOLECULE'S DISTRIBUTION}

In this appendix we are going to show how the eigenvalues and eigenvectors of the general Hamiltonian (17) with different distribution of molecules are related to those of the simple asymmetric exclusion Hamiltonian (1). Let us consider initially the case of free boundary conditions. In this case we have to specify, for a given lattice size $N$, the occupation at the border of the lattice, i. e., the minimal $x_{I}$ and maximum $x_{F}$ coordinates we may put a molecule of size $s$. As an extension of (2) we define these coordinates, for the model with a distribution $\left\{s_{1}, s_{2}, \ldots, s_{n}\right\}$ of molecules as

$$
x_{I}=d_{s_{1}, 0}+\delta_{s_{1}, 0}=\operatorname{Int}\left[\left(s_{1}+1\right) / 2\right]+\delta_{s_{1}, 0}, \quad x_{F}=N-\left(d_{s_{n}, 0}-1\right)-\delta_{s_{n}, 0} .
$$

If $\mid x_{1}, \ldots, x_{n}>$ are the vectors corresponding to the coordinates of the particles of sizes $\left\{s_{1}, \ldots, s_{n}\right\}$ the application of the Hamiltonian (7) with free ends in a given vector can be written as

$$
\begin{aligned}
& H_{\{s\}}^{F} \mid x_{1}, \ldots, x_{n}>= \\
& \quad-\sum_{i=1}^{n-1} \epsilon_{+} \theta\left(x_{i+1}-x_{i}-d_{s_{i}, s_{i+1}}\right)\left[\left|x_{1}, \ldots, x_{i-1}, x_{i}+1, x_{i+1}, \ldots, x_{n}>-\right| x_{1}, \ldots, x_{n}>\right] \\
& \quad-\sum_{i=2}^{n} \epsilon_{-} \theta\left(x_{i}-x_{i-1}-d_{s_{i-1}, s_{i}}\right)\left[x_{1}, \ldots, x_{i-1}, x_{i}-1, x_{i+1}, \ldots, x_{n}>-\mid x_{1}, \ldots, x_{n}>\right]
\end{aligned}
$$




$$
\begin{aligned}
& -\epsilon_{+} \theta\left(x_{F}-x_{n}\right)\left[\left|x_{1}, \ldots, x_{n-1}, x_{n}+1>-\right| x_{1}, \ldots, x_{n}>\right] \\
& -\epsilon_{-} \theta\left(x_{1}-x_{I}\right)\left[\left|x_{1}-1, x_{2}, \ldots, x_{n}>-\right| x_{1}, \ldots, x_{n}>\right],
\end{aligned}
$$

where $\theta(x)=0$ for $x \leq 0$ and $\theta(x)=1$ for $x>0$, is the standard step function.

If we now make the following change of coordinates

$$
x_{i}^{\prime}=x_{i}-x_{I}-\sum_{j=1}^{i-1} d_{s_{j}, s_{j+1}}+i
$$

we can rewrite (A1) as

$$
\begin{aligned}
& H_{\{s\}}^{F} \mid x_{1}^{\prime}, \ldots, x_{n}^{\prime}>= \\
& \quad-\sum_{i=1}^{n-1} \epsilon_{+} \theta\left(x_{i+1}^{\prime}-x_{i}^{\prime}-1\right)\left[\left|x_{1}^{\prime}, \ldots, x_{i-1}^{\prime}, x_{i}^{\prime}+1, \ldots, x_{n}^{\prime}>-\right| x_{1}^{\prime}, \ldots, x_{n}^{\prime}>\right] \\
& \quad-\sum_{i=2}^{n} \epsilon_{-} \theta\left(x_{i}^{\prime}-x_{i-1}^{\prime}-1\right)\left[\left|x_{1}^{\prime}, \ldots, x_{i-1}^{\prime}, x_{i}^{\prime}-1, x_{i+1}^{\prime}, \ldots, x_{n}^{\prime}>-\right| x_{1}^{\prime}, \ldots, x_{n}^{\prime}>\right] \\
& \quad-\epsilon_{+} \theta\left(x_{F}^{\prime}-x_{n}^{\prime}\right)\left[\left|x_{1}^{\prime}, \ldots, x_{n-1}^{\prime}, x_{n}^{\prime}+1>-\right| x_{1}^{\prime}, \ldots, x_{n}^{\prime}>\right] \\
& \quad-\epsilon_{-} \theta\left(x_{1}^{\prime}-x_{I}^{\prime}\right)\left[\left|x_{1}^{\prime}-1, x_{2}^{\prime}, \ldots, x_{n}^{\prime}>-\right| x_{1}^{\prime}, \ldots, x_{n}^{\prime}>\right],
\end{aligned}
$$

where now $x_{I}^{\prime}=1$ and $x_{F}^{\prime}=x_{F}-x_{I}-\sum_{j=1}^{n-1} d_{s_{j}, s_{j+1}}+n \equiv N^{\prime}$. But this is exactly the application of the simple asymmetric exclusion Hamiltonian $H_{\left\{s_{1}=\cdots=s_{n}=1\right\}}^{F}$ in a lattice size $N^{\prime}$. Consequently for free ends there exists a one-to-one correspondence between the eigenvalues and eigenvectors of our general Hamiltonian (7) with arbitrary distribution of particle's sizes with that of the standard simple diffusion problem, in a lattice size which depends on the volume excluded due to the molecule's sizes, i. e.,

$$
\begin{array}{r}
H_{\{s\}}^{F}(n, N)=H_{\left\{s_{1}=s_{2}=\cdots=s_{n}=1\right\}}^{F}\left(n, N^{\prime}\right) \\
N^{\prime}=N-\sum_{j=1}^{n-1} d_{s_{j}, s_{j+1}}-d_{s_{1}, 0}-d_{s_{n}, 0}+n+1-\delta_{s_{1}, 0}-\delta_{s_{n}, 0} .
\end{array}
$$

The Bethe ansatz solution of the XXZ chain with surface fields given in [20], after the canonical transformation (11) can be easily exported for our general model (7) with free ends. As observed in [21] the simple asymmetric diffusion Hamiltonian (1), with free boundaries, has a quantum $U_{q} S U(2)$ symmetry with $q=\sqrt{\frac{\epsilon_{+}}{\epsilon_{-}}}$. This symmetry implies an exact form for 
the ground-state wavefunction. Using this wavefunction in the relation (A3) we obtain the ground state wavefunction for the general Hamiltonian (7) with free ends

$$
\left.\Psi_{0}^{\{s\}}=\sum_{\{x\}} \prod_{i=1}^{n}\left(\frac{\epsilon_{+}}{\epsilon_{-}}\right)^{x_{i}+i-\sum_{j=1}^{i-1} d_{s_{j}, s_{j+1}}}\right) \mid x_{1}, x_{2}, \ldots, x_{n}>.
$$

Let us now consider the case of the Hamiltonian (7) with twisted boundary conditions specifyed by the angle $\phi$, which in general is a complex number,

$$
E_{N+1}^{\beta, 0}=e^{i \phi} E_{1}^{\beta, 0}, \quad E_{N+1}^{0, \beta}=e^{-i \phi} E_{1}^{0, \beta}, \quad \beta \neq 0 E_{n+1}^{0,0}=E_{1}^{0,0}, \quad E_{N+1}^{\beta, \beta}=E_{1}^{\beta, \beta} .
$$

The periodic case treated in section (3) corresponds to the case where $\phi=0$. The Betheansatz equations for these boundary conditions can be obtained by changing (52) and (53), and are given by

$$
e^{i k_{j} N^{\prime}} e^{i \tilde{\phi}_{m}}=(-1)^{n-1} \prod_{l=1}^{n} \frac{\epsilon_{+}+\epsilon_{-} e^{i\left(k_{j}+k_{l}\right)}-e^{i k_{j}}}{\epsilon_{+}+\epsilon_{-} e^{i\left(k_{j}+k_{l}\right)}-e^{i k_{l}}},
$$

where

$$
\begin{array}{r}
N^{\prime}=N-n(\tilde{s}-1), \quad \tilde{\phi}_{m}=\phi+P(\tilde{s}-1)+\frac{2 \pi}{r} m, \quad m=0,1, \ldots, r-1, \\
P=\sum_{j} k_{j} \quad \bmod (2 \pi)=\frac{2 \pi}{N} l, \quad l=0,1, \ldots, N-1,
\end{array}
$$

is the momentum and $r, \tilde{s}$ are defined in (57)-(60). These equations give us the following equivalence between the eigenspectra of the general Hamiltonian (7) with boundary condition $\phi$, in the eigensector with $n$ particles and a given momenta $P$

$$
H_{\left\{s_{1}, \ldots, s_{n}\right\}}^{\phi}(N, P, n)=\sum_{m=0}^{r-1} H_{\left\{s_{1}=s_{2}=\cdots=s_{n}=1\right\}}^{\tilde{\phi}_{m}}\left(N^{\prime}, P, n\right),
$$

where in the right-hand side we have the eigenspectra of the asymmetric simple exclusion Hamiltonian (1) with twisted boundary condition $\tilde{\phi}_{m}$. In the right-hand side of the above equation we have also to add several eigenspectra, depending on the value of $r$ (see Sec. 3), and this is due to the distinguibility of the particles in the Hamiltonian of the left-hand side of the equation. 
Our Bethe solutions presented in Sec. 3 also give us a connection between the wavefunctions of the models. The eigenstates related by (A12), apart from an overall normalization satisfy

$$
\Psi_{\left\{s_{1}, \ldots, s_{n}\right\}}^{\left\{k_{1}, \ldots, k_{n}\right\}}\left(x_{1}, x_{2}, \ldots, x_{n}\right)=\Psi_{\left\{s_{1}+s_{2}=\cdots=s_{n}=1\right\}}^{\left\{k_{1}, \ldots, k_{n}\right\}}\left(x_{1}^{\prime}, x_{2}^{\prime}, \ldots, x_{n}^{\prime}\right)
$$

where

$$
x_{1}^{\prime}=x_{1}, \quad x_{i}^{\prime}=x_{i}+i-1-\sum_{j=1}^{i-1} d_{s_{j}, s_{j+1}}, \quad i=2, \ldots, n .
$$

The results (A5) and (A13)-(A14) imply that any calculation involving only wavefunctions can be straigtforwardly translated for arbitrary distribution $\{s\}$ of molecule's sizes. An example of this is the $n$-particle Green's function $P^{\{s\}}\left(x_{1}, \ldots, x_{n} ; t \mid y_{1}, \ldots, y_{n} ; 0\right)$ which gives the probability of finding particles of size $s_{i}$, initially $(t=0)$ at $y_{i}$ and at time $t$ at $x_{i}$ $(i=1, \ldots, n)$. These Green's functions for the different models satisfy

$$
P^{\left\{s_{1}, \ldots, s_{n}\right\}}\left(x_{1}, \ldots, x_{n} ; t, y_{1}, \ldots, y_{n} ; 0\right)=P^{\left\{s_{1},=\cdots=s_{n}=1\right\}}\left(x_{1}^{\prime}, \ldots, x_{n}^{\prime} ; t, y_{1}^{\prime}, \ldots, y_{n}^{\prime} ; 0\right)
$$

where $x_{i}^{\prime}$ and $y_{i}^{\prime}$ are related to $x_{i}$ and $y_{i}$ as in (A3). The above result generalizes that obtained by Sasamoto and Wadati [12] for the case we have a fully asymmetric model $\left(\epsilon_{-}=0\right)$ and molecules of identical sizes $s_{1}=s_{2}=\cdots=s_{n}=s$.

Calculation involving eigenvalues, like the calculation of the exponent $z$ we did in Sec. 4 for the fully asymmetric model $\left(\epsilon_{-}=0\right)$ should be translated with care among the different models. The Bethe-ansatz equations ( (17) in a periodic lattice of size $N(\phi=0)$ are the same as those of the simple exclusion Hamiltonian (1) in a lattice of size $N^{\prime}=N-n(\tilde{s}-1)$ and twisted boundary condition $\tilde{\phi}_{m}=P(\tilde{s}-1)+\frac{2 \pi}{r} m, m=0,1, \ldots, r-1$. However the eigenvalues of the simple asymmetric exclusion Hamiltonian (11) depend on the boundary condition. Our results of Sec. 3 although valid only for $\epsilon_{-}=0$ indicate that the effect of the boundary angle in the finite-size corrections is of higger order than the leading corrections for the first excited state, since in this case $\tilde{\phi}_{m}=\tilde{\phi}_{0}=2 \pi(\tilde{s}-1) / N$. This imply that for arbitrary distribution of molecules 
and arbitrary values of $\epsilon_{+}, \epsilon_{-}$and densities we can use the results obtained in [10], for the leading order of the real part of the mass gap, which gives a universal dynamical critical exponent $z=\frac{3}{2}$ of KPZ type. 


\section{REFERENCES}

[1] See e. g., T. Ligget, Interacting Particle Systems (Springer-Verlag, Berlin, 1985).

[2] D. Kim, J. Phys. A 30, 3817 (1997).

[3] G. Albertini, S. R. Dahmen, and B. Wehefritz, Nucl. Phys. B 493, 541 (1997).

[4] S. R. Dahmen, B. Wehefritz, and G. Albertini, preprint cond-mat/9802152.

[5] P. Meakin, P. Ramanlal, L. M. Sander, and R. C. Ball, Phys. Rev. A 34, 5091 (1986).

[6] D. Liu and M. Plischke, Phys. Rev. B 38, 4781 (1988).

[7] L. H. Gwa and H. Spohn, Phys. Rev. Lett. 68, 725 (1992), Phys. Rev. A 46, 844 (1992).

[8] M. Kardar, G. Parisi, and Y.C. Zhang, Phys. Rev. Lett. 56, 889 (1986).

[9] For a review see, J. Krug and H. Spohn, in Solids Far From Equilibrium: Growth, Morphology and Defects, edited by C. Godréche (Cambridge University Press, Cambridge, England, 1991).

[10] D. Kim, Phys. Rev. E 52, 3512 (1995).

[11] F. C. Alcaraz and R. Z. Bariev, Phys. Lett. A 240247 (1998).

[12] T. Sasamoto and M. Wadati, J. Phys. A 31, 6057 (1998).

[13] F. C. Alcaraz, M. Droz, M. Henkel, and V. Rittenberg, Ann. Phys. (N.Y.) 230, 250 (1994).

[14] M. Henkel and G. M. Schütz, Physica (Amsteram) 206, 187 (1994).

[15] F. C. Alcaraz, M. N.Barber, and M. T. Batchelor, Ann. Phys. (N.Y.) 182, 280 (1988).

[16] N. M. Bogoliubov and T. Nassar, Phys. Lett. A 234, 345 (1997).

[17] N. M. Bogoliubov, A. G. Izergin, and N. A. Kitanine, Nucl. Phys. B 516, 501 (1998).

[18] C. N. Yang, Phys. Rev. Lett. 19, 1312 (1967). 
[19] R. J. Baxter, Exactly Solved Models in Statistical Mechanics (Academic Press, New York, 1982).

[20] F. C. Alcaraz, M. N. Barber, M.T. Batchelor, R. J. Baxter, and G. R. W. Quispel, J. Phys. A 20, 6397 (1987).

[21] F. C. Alcaraz and V. Rittenberg, Phys. Lett. B 324, 377 (1993). 


\section{Figure Captions}

Figure 1 - Example of configurations of molecules with distinct sizes in the asymmetric diffusion problem. These examples correspond to $n=5$ particles in a lattice with size $N=6$. The sizes $\left\{s_{1}, s_{2}, \ldots, s_{5}\right\}$ are shown in figures (a)-(c).

Figure 2 - Examples of configurations in the growth model with $N=7, b=3$ and $n=2$. The corresponding configurations of particles in the asymmetric diffusion problem (Eq. (77))

are also shown. The configurations $(\mathrm{b})$ and $(\mathrm{d})$ are obtained by adsorbing a brick (size $b=3$ ) or by moving a particles to the left in configuration (a), and configuration (c) and (e) are obtained by desorbing a brick, or by moving a particle to the right in configuration (a).

\section{Table Caption}

Table 1 - Examples of finite-size estimates for the amplitudes $a_{0}, b_{0}$ and the exponents $z$ and $\gamma$ in (73). These estimates correspond to the cases $\tilde{s}=0.25$ and $\tilde{s}=2.5$. The calculated analitical results in the $n \rightarrow \infty$ are also shown in the last line of this table. 


\section{Table 3}

\begin{tabular}{|c|c|c|c|c|c|c|c|c|}
\hline$n$ & \multicolumn{5}{|c|}{$\tilde{s}=0.25$} & \multicolumn{4}{c|}{$\tilde{s}=2.5$} \\
\hline & $a_{0}$ & $b_{0}$ & $z$ & $\gamma$ & $a_{0}$ & $b_{0}$ & $z$ & $\gamma$ \\
\hline 10 & 2.522601 & -0.916488 & 1.586388 & 0.934332 & 2.495387 & 0.654527 & 1.576255 & 0.934156 \\
\hline 50 & 2.351517 & -0.941304 & 1.521414 & 0.997428 & 2.345566 & 0.672351 & 1.518526 & 0.997403 \\
\hline 100 & 2.326504 & -0.942187 & 1.510838 & 0.999374 & 2.323540 & 0.672989 & 1.509564 & 0.999367 \\
\hline 150 & 2.318133 & -0.942349 & 1.507253 & 0.999724 & 2.316160 & 0.673106 & 1.506402 & 0.999721 \\
\hline 200 & 2.313942 & -0.942406 & 1.505450 & 0.999846 & 2.312463 & 0.673146 & 1.504811 & 0.999844 \\
\hline 250 & 2.311425 & -0.942432 & 1.504365 & 0.999902 & 2.310243 & 0.673165 & 1.503853 & 0.999900 \\
\hline 300 & 2.309747 & -0.942446 & 1.503640 & 0.999932 & 2.308737 & 0.673175 & 1.503189 & 0.999957 \\
\hline 400 & 2.307648 & -0.942460 & 1.502733 & 0.999962 & & & & \\
\hline 500 & 2.306388 & -0.942466 & 1.502186 & 0.999976 & & & & \\
\hline 800 & 2.304498 & -0.942473 & 1.501364 & 0.999988 & & & & \\
\hline \hline EXACT 2.301346 & -0.942478 & 1.5 & 1 & 2.301346 & 0.673198 & 1.5 & 1 \\
\hline
\end{tabular}


a)
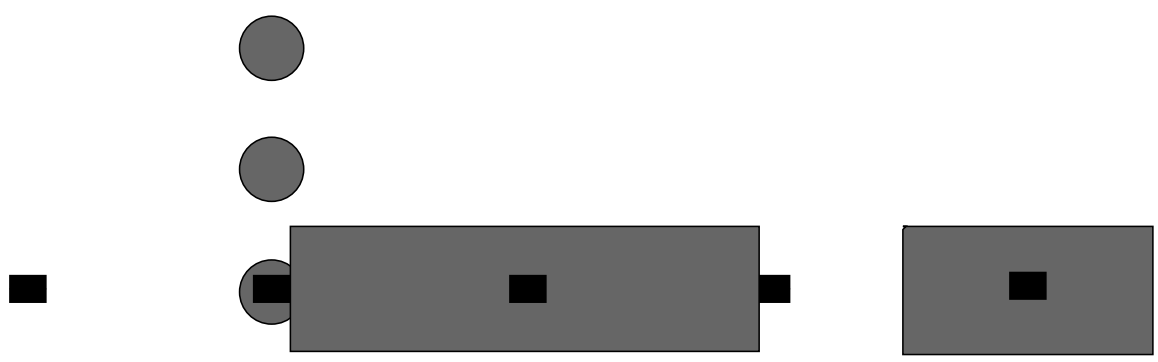

$$
\{s\}=\{0,0,0,2,1\}
$$

b)

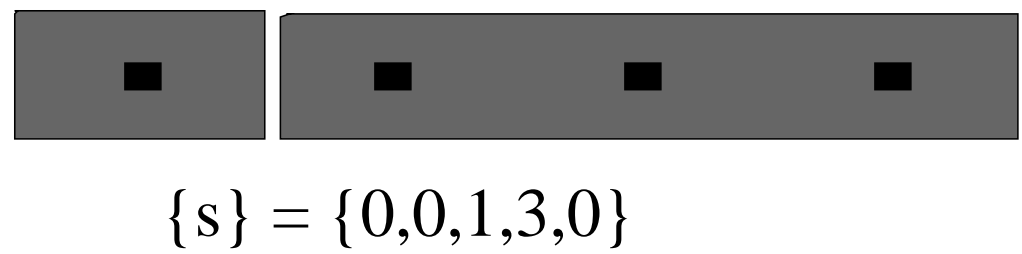

0

c)

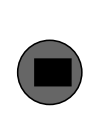

$\{s\}=\{0,0,1,3,0\}$

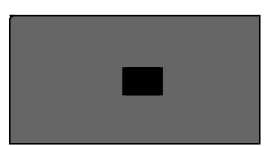

0
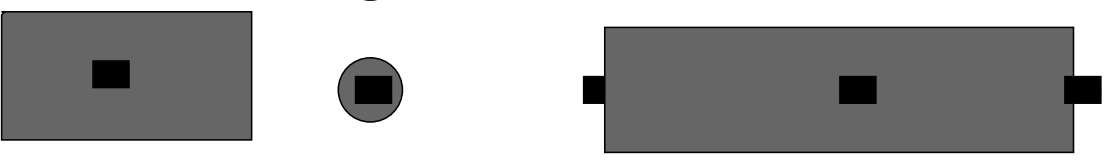

$\{s\}=\{1,1,0,0,2\}$ 


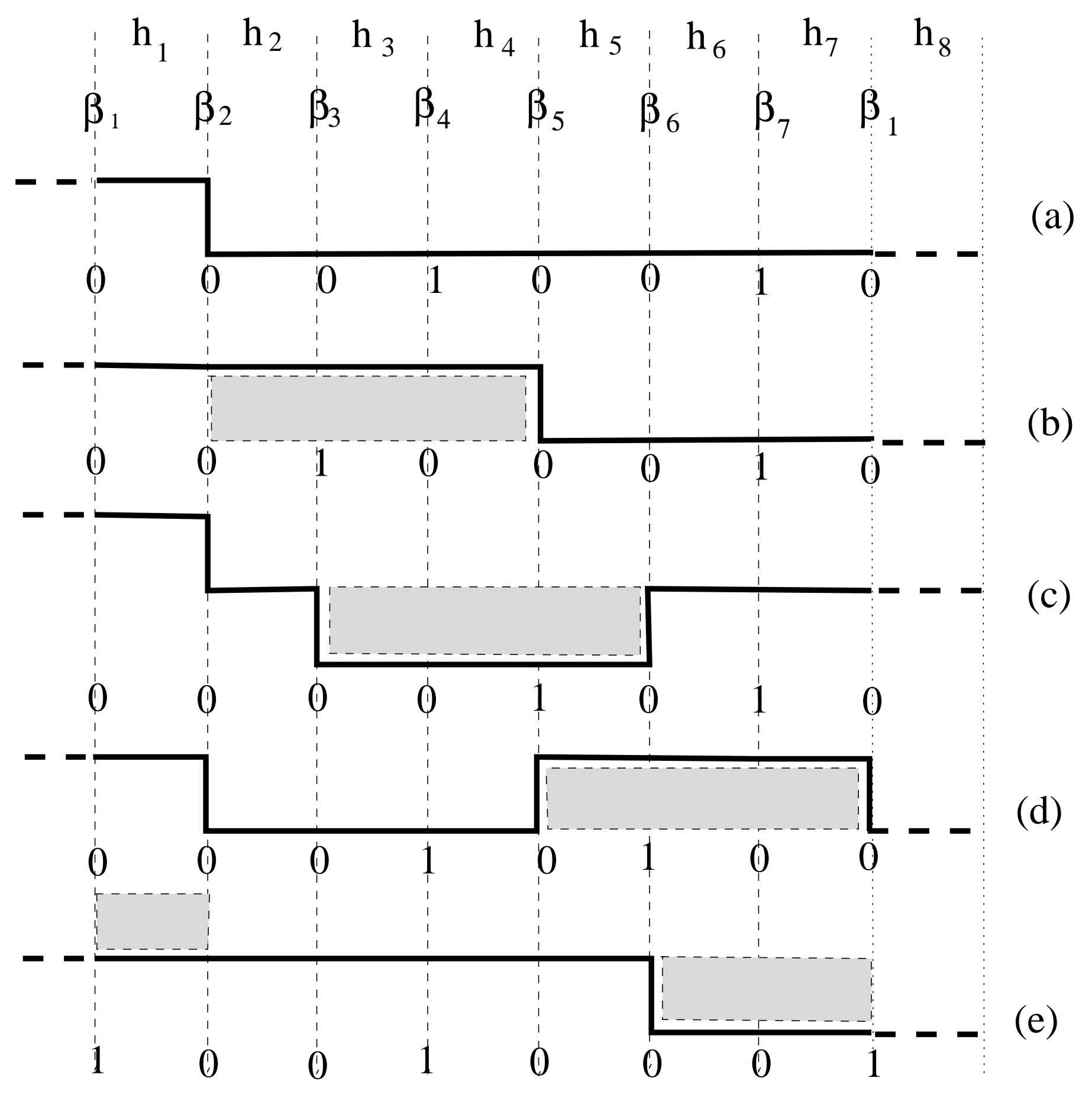

